\section{Estudos de consumo alimentar: aspectos metodológicos gerais e o seu emprego na avaliação de crianças e adolescentes}

\section{Food consumption studies: general methodological aspects and its use in the evaluation of children and adolescents aged}

Ana Augusta Monteiro Cavalcante 1

Silvia Eloiza Priore 2

Sylvia do Carmo Castro Franceschini 3

1-3 Departamento de Saúde e Nutrição.

Universidade Federal de Viçosa.

Av. P. H. Rolfs, s. n. Viçosa, MG, Brasil. CEP: 36.571-000

\begin{abstract}
This article discusses methodological aspects of food consumption studies in children and adolescents. To reach the understanding of these methods it provides the value of Nutritional Epidemiology in a general context through a descriptive study of diet relation with the manifestation of disease as well as historical origin, value, advantage, constraints and use of methods employed to measure food intake, especially in children and adolescents in population epidemiological studies. In addition, it presents classic and contemporaneous literature demonstrating results obtained and some suggestions to improve these methods. Finally, it discloses the more relevant aspects that by consensus of many researchers compete with constraining and facilitating factors in their use providing them with more validity and reproducibility concerning studies of food consumption for children and adolescents.
\end{abstract}

Key words Food consumption, Diet inquiries, Children, Adolescents
Resumo Este artigo discute aspectos metodológicos dos estudos de consumo alimentar em crianças e adolescentes. Para compreender esses aspectos, descreve, em contexto mais amplo, a importância da Epidemiologia Nutricional no estudo descritivo da relação da dieta com o surgimento das enfermidades, bem como origem histórica, importância, vantagens, limitações e usos dos métodos empregados para medir a ingestão alimentar, especialmente em crianças e adolescentes, em estudos epidemiológicos populacionais. Apresenta ainda alguns trabalhos da literatura científica clássica e contemporânea, demonstrando os resultados obtidos e algumas sugestões para aperfeiçoamento desses métodos. Finalmente, evidencia os aspectos mais relevantes que, por consenso de vários pesquisadores, competem como fatores limitantes ou facilitadores no emprego desses métodos, conferindo a esses, maior validade e reprodutibilidade nos estudos de consumo alimentar de crianças e adolescentes.

Palavras-chave Consumo alimentar, Inquéritos sobre dietas, Criança, Adolescente 


\section{Introdução}

A avaliação do consumo alimentar tem um papel crítico na área de pesquisa em nutrição e saúde e também no desenvolvimento de programas. Entretanto, para avaliar o consumo alimentar, são necessários métodos apropriados para estimar a ingestão de alimentos e nutrientes de grupos populacionais. ${ }^{1}$

A complexidade da dieta humana tem instigado pesquisadores a procurar os meios mais adequados para avaliar qualitativa e quantitativamente o consumo de alimentos, dimensionar a adequação de nutrientes e relacionar dieta à ausência de saúde. ${ }^{2}$

Para pesquisadores como Margetts e Nelson, 2 Majem,3 Bonomo,4 Fisberg et al.,5 Villar,6 Nowak e Büttner, ${ }^{7}$ os dados sobre consumo de alimentos são coletados com diversos propósitos e, os mais relevantes para a epidemiologia nutricional são estimar a adequação da ingestão dietética de grupos populacionais, investigar a relação entre dieta, saúde e estado nutricional e avaliar a educação nutricional, a intervenção nutricional e os programas de suplementação alimentar.

Fatores como complexidade da dieta, hábitos alimentares, qualidade da informação, idade, imagem corporal, memória do entrevistado, crenças, comportamento, cultura e status socioeconômico, bem como fatores de exposição, são variáveis que interferem e tornam muito difícil o ato de registrar a ingestão de um indivíduo, sem exercer influência sobre esse.2,5

Até a Segunda Guerra Mundial, pouca atenção se dava ao desenvolvimento de estimativas de oferta e consumo de alimentos. Foi a preocupação dos governos com a guerra, a escassez de alimentos e o aumento do controle da distribuição de alimentos que contribuíram para o aprimoramento dessas estatísticas. Cada país passou a ter necessidade de conhecer sua capacidade de produzir e estocar alimentos e, também, a de outros povos, especialmente dos seus inimigos. 8

Posteriormente, grandes progressos metodológicos foram alcançados e um grande número de indicadores de consumo alimentar pôde ser construído. 8

Relatos de Coimbra et al. 9 descrevem os primeiros inquéritos nutricionais realizados no Brasil, na década de 30, por Josué de Castro, sob a influência de Pedro Escudero, em crianças e pré-escolares oriundos de 500 famílias da classe operária de Recife.

Pesquisas de consumo de alimentos constituem instrumentos eficazes e de baixo custo para obtenção de informações sobre as características de consumo alimentar de grande parte da população; entretanto, devido ao uso e as limitações de cada método, a escolha do instrumento para medir a informação dietética não constitui tarefa fácil.4

A medição da informação dietética através das pesquisas de consumo alimentar representa, portanto, um grande avanço que possibilita fazer levantamentos mais precisos da ingestão de alimentos e a conversão dessas quantidades em calorias, macro e micronutrientes. 10

Várias metodologias vêm sendo utilizadas para avaliar o consumo dietético de indivíduos em estudos epidemiológicos, no sentido de obter dados válidos, reprodutíveis e comparáveis. Dentre estes métodos se destacam o questionário de freqüência alimentar, o recordatório 24 horas, o método do inventário, o registro diário ou diário alimentar, e a história dietética. Entretanto, cada método tem suas vantagens e desvantagens. 4,11

Em outra via, apesar dos importantes avanços conquistados nos últimos anos no campo da epidemiologia nutricional, a ausência de instrumentos validados e reprodutíveis, que possibilitem a medição com confiança e precisão, constitui-se uma das maiores dificuldades que persiste ao se estudar a relação dieta/doença.2,12

As evidências têm demonstrado que, para determinado método dietético ser válido, a informação mensurada deverá refletir exatamente o que pretende medir; assim, os resultados poderão ser generalizados de maneira eficaz. 2,12-15

O consumo alimentar vem sendo estudado devido a potencial relação com as doenças crônicas nãotransmissíveis. O questionário de freqüência semiquantitativa de alimentos é especialmente usado para descrever essa relação em muitos estudos epidemiológicos. 16 Por uma série de fatores, que serão discutidos mais adiante neste artigo, a sua avaliação acurada é um dos mais difíceis aspectos da abordagem nutricional.

Majem $^{3}$ afirma que a correta avaliação do consumo alimentar é influenciada por fatores como a complexidade da dieta e a ingestão ou consumo verdadeiro do indivíduo ou de uma coletividade. Na sua opinião, a avaliação da dieta torna-se complexa e, muitas vezes, difícil em razão de os conceitos relativos à dieta dos indivíduos serem mal interpretados. A medida da ingestão ou consumo verdadeiro de um indivíduo ou coletividade não é igual ao seu consumo habitual. As diferenças derivam da forma como os métodos colhem e mensuram a informação dietético-nutricional. A medida da variabilidade da dieta, os hábitos e fatores de exposição são variáveis que interferem e tornam muito difícil o ato de registrar a ingestão de um indivíduo, sem exercer influência sobre esse. $.3,4$ 
Para Nelson e Margetts (1997:133):2 "... virtualmente todos os métodos dependem da habilidade do indivíduo para fornecer informações exatas e ainda, estas informações precisam ser validadas ...". O propósito do processo de validação é avaliar a extensão para a qual os indivíduos podem ser classificados erroneamente, usando-se as medidas instrumentais escolhidas.

Com base nos conhecimentos sobre os métodos que permitem a obtenção de dados sobre ingestão dietética, seus usos e limitações, o presente trabalho tem como objetivo analisar os aspectos metodológicos dos estudos de consumo alimentar em crianças e adolescentes.

\section{Abordagem histórica dos inquéritos dietéticos}

No Século XIX, a maioria dos estudos de consumo alimentar de populações tinha por finalidade estabelecer padrões dietéticos, que, em sua maior parte, eram levantamentos dietéticos. As recomendações de nutrientes e energia foram baseadas nesses levantamentos. 8,17

Um século mais tarde, os estudos sobre consumo alimentar evoluíram e passaram a ser realizados, em muitos países, por organismos oficiais, com o objetivo de estabelecer as recomendações de energia e demais nutrientes e orientar as políticas governamentais no campo da Saúde Pública, sobretudo nos programas de fortificação de alimentos, suplementação alimentar e educação nutricional para as populações.

$\mathrm{O}$ primeiro estudo internacional consistiu em comparar sistematicamente a oferta nacional de alimentos e as variações observadas no consumo entre Estados Unidos, Canadá e Inglaterra. Assim, o relatório produzido pelo "Combined Food Broad" em 1944 buscou orientar a alocação internacional de alimentos. 8

Ainda no século XX, o segundo grande marco para os estudos de consumo alimentar foi o "World Food Survey", publicado em 1946 pela Food and Agriculture Organization (FAO) que teve a função de compilar estimativas de oferta de alimentos de mais de 70 países antes da Guerra, por meio do método de balanço de alimentos, que identifica a disponibilidade de alimentos para consumo, comparando a produção às diferentes utilizações dos alimentos existentes em um país. Refletem, portanto, a disponibilidade de alimentos. 8,18

Esses documentos foram muito criticados porque não corresponderam às expectativas de servirem como parâmetro para decisões políticas, além de terem suscitado críticas as suas estimativas da extensão e distribuição geográfica da fome mundial. Entretanto, tiveram papel relevante ao despertar a atenção de pesquisadores, governos e organismos internacionais sobre os diferentes padrões de consumo alimentar característicos dos diferentes países, estimulando os governos a avaliar e aprimorar a qualidade de suas estatísticas alimentares no decorrer dos anos. 8

No Brasil, os primeiros relatos de pesquisa de consumo alimentar de populações são da década de 30. Coimbra et al. 9 e Vasconcelos 19 relatam a pesquisa realizada por Josué de Castro com famílias da classe operária do município de Recife, Pernambuco, que se baseou na metodologia de orçamento e padrão de consumo alimentar. A pesquisa, intitulada "Condições de vida das classes operárias no Recife", evidenciou um regime alimentar de péssima qualidade nutricional e a incapacidade do mesmo de suprir as necessidades energéticas desse grupo populacional.

Salvo e Gimeno ${ }^{20}$ ressaltam que foi durante a década de 60 , período marcado por grandes mudanças sociais e surgimento de uma consciência global e doméstica da fome e das questões de saúde, que foram conduzidos os primeiros estudos epidemiológicos com grande número de indivíduos, na tentativa de estabelecer uma ligação entre dieta e aparecimento de doenças.

Em relatos de Vasconcelos 19 encontra-se a descrição histórica do primeiro convênio firmado entre o Instituto de Nutrição da Universidade Federal de Pernambuco e o Interdepartmental Committee on Nutrition for National Development (ICNND), dos Estados Unidos, em 1962, para realização de uma investigação nutricional do Nordeste do Brasil. Tratava-se de um levantamento populacional denominado "Northeast Brazil survey", cuja metodologia consistiu na aplicação de inquéritos dietéticos (recordatório 24 horas e folhas de balanço de alimentos), exames clínicos nutricionais e exames laboratoriais. A população examinada foi composta por 5009 indivíduos de todas as faixas de idade, dos quais 875 eram crianças menores de cinco anos.

Para Batista Filho, relatado por Vasconcelos (2001: 76),19 essa pesquisa "... quantificou no Nordeste brasileiro, deficiências alimentares de grande relevância para a saúde pública, como a desnutrição e a hipovitaminose A, mas tinha falhas na metodologia, principalmente na amostra, que era pequena..."

$\mathrm{Na}$ opinião de Galeazzi (2000: 71),21 ".. a análise de consumo alimentar de base populacional no Brasil se pauta em três estudos realizados pelo IBGE, em 1974/75 - ENDEF (Estudo Nacional da 
Despesa Familiar) e as POFs (Pesquisas de Orçamentos Familiares), em 1987 e 1996 ... "; entretanto aponta para as possíveis aplicações e limitações da metodologia utilizada para medir as informações nutricionais, chamando a atenção para a escolha correta da base de dados e os perfis nutricionais, que devem ser provenientes de metodologias que se proponham a investigar o consumo alimentar de forma acurada.

O ENDEF, pesquisa domiciliar de abrangência nacional que coletou dados sobre os orçamentos familiares e o consumo de alimentos em 55.000 domicílios, cobrindo quase todo o território nacional, com exceção das áreas rurais das macrorregiões Norte e Centro-Oeste, foi concebido com objetivos múltiplos, dentre os quais se destaca a análise da situação nutricional das famílias. 10

A metodologia de pesquisa aplicada no ENDEF consistia em visitar cada domicílio ao longo de sete dias consecutivos, para captar as diferenças de ritmos alimentares entre os dias úteis e o fim de semana, por meio do método da pesagem dos alimentos. 10,22

As séries históricas dos estudos do ENDEF e POFs são importantes dados para as informações das tendências, sempre procurando validá-las com outras bases de informação. ${ }^{21}$

Ainda hoje, informações sobre o consumo alimentar da população brasileira são baseadas na fonte de dados proveniente do ENDEF. Pesquisas mais recentes como as POFs, a Pesquisa Nacional sobre Saúde e Nutrição 23 foram importantes, mas não tiveram enfoque no consumo alimentar. ${ }^{21}$

Entretanto, o Brasil apresentou mudanças profundas no seu perfil socioeconômico nos últimos decênios que se seguiram ao ENDEF e fez-se urgente o conhecimento do perfil alimentar da população brasileira para nortear as políticas públicas na área de alimentação e nutrição. 24

No final da década de 90, uma parceria do Ministério da Saúde com algumas universidades brasileiras permitiu a realização do "Estudo multicêntrico sobre consumo alimentar" 24 que levantou dados sobre o consumo alimentar de cinco cidades brasileiras: Rio de Janeiro, Goiânia, Campinas, Ouro Preto e Curitiba. A metodologia aplicada nesse estudo consistiu na aplicação de dois inquéritos, o Inquérito de Consumo Familiar e o Inquérito de Consumo Individual, que permitiram, não somente, conhecer o perfil alimentar e nutricional da população brasileira, mas também, nortear o planejamento das ações do governo com respeito à carência alimentar e a distribuição e abastecimento de alimentos.

Apesar da escassez de estudos nacionais mais abrangentes, alguns estudos regionais têm procurado preencher a lacuna de conhecimento existente com relação ao consumo alimentar de grupos populacionais, especialmente crianças e adolescentes.25-30

\section{Metodologia de consumo alimentar}

Os métodos dietéticos têm o objetivo de medir a informação dietética e podem ser classificados em qualitativos e quantitativos. ${ }^{31-33}$ Marr32 simplifica a classificação dos métodos definindo-os em duas categorias: os que registram o consumo atual de alimentos (pesagem de alimentos, registro alimentar e o recordatório 24 horas) e os que recordam o consumo passado de alimentos (história dietética e questionário de consumo alimentar).

Para Bingham, 34 as técnicas de avaliação dietética, provenientes de registros de peso de alimentos, questionários de consumo alimentar e marcadores biológicos, vêm sendo criticamente revisadas com o objetivo de avaliar a exatidão de cada método.

Preocupados com o surgimento de muitos métodos e o crescente interesse em estudar a relação causaefeito de doenças em estudos epidemiológicos, muitos pesquisadores se especializaram no questionário de freqüência de consumo alimentar. ${ }^{34-36}$

A FAO tem reconhecido a necessidade de avaliar o consumo alimentar e o estado nutricional de populações para desenvolver programas de saúde e nutrição. 37

A escolha do método deve fundamentar-se nos objetivos da pesquisa ou no tipo de estudo, além de considerar os recursos disponíveis. Fundamental também é a escolha dos instrumentos de avaliação do consumo, que devem conferir validade e reprodutibilidade, além de caracterizarem fielmente a dieta do indivíduo. 16

Segundo considerações de alguns autores, a escolha do método demonstra o desafio que se impõe aos pesquisadores na avaliação correta do consumo alimentar em estudos epidemiológicos.2,4.16

$\mathrm{Na}$ epidemiologia nutricional descritiva, os estudos transversais e ecológicos, permitem o conhecimento da dieta atual dos indivíduos e, na epidemiologia nutricional analítica, os estudos de caso-controle e de coorte possibilitam que se conheça a dieta habitual. Nos estudos de coorte, caracterizados por serem estudos de segmento que demandam mais tempo, o trabalho metodológico requer maiores cuidados. 4

Além disso, para avaliar a dieta atual e habitual em estudos epidemiológicos podem-se utilizar outras abordagens, como testes bioquímicos e dimensões ou composição corporal. Dentre estas abordagens, Willett ${ }^{36}$ considera que a medida das dimensões ou 
composição corporal reflete os efeitos da dieta em períodos mais prolongados.

Os métodos utilizados para avaliar o consumo alimentar comumente encontram-se conjugados entre si ou associados a outros parâmetros de avaliação do estado nutricional de indivíduos. Essa junção pode propiciar melhor compreensão e interpretação dos resultados obtidos em determinado estudo. $37-40$

Salvo e Gimeno 20 consideram que os métodos para avaliar o consumo alimentar vêm evoluindo ao longo do tempo, o que evidencia as limitações das metodologias empregadas diante das necessidades de avaliar o hábito alimentar dos indivíduos.

\section{Metodologia de consumo alimentar aplicada a crianças e adolescentes}

Em documento científico, o Comitê de Expertos da FAO, WHO e UNU (Universidade das Nações Unidas) ${ }^{41}$ para Nutrição Humana chama atenção para as dificuldades de se medir a ingestão de alimentos de crianças e adolescentes. Segundo esse Comitê, os critérios mais importantes a considerar na escolha do método para coletar dados sobre a ingestão de alimentos de crianças e adolescentes são: que a técnica não interfira nos hábitos dietéticos, que os dados possam ser representativos da dieta usual ou habitual e que a técnica possa ser preferencialmente, aplicada em estudos de grupos.

Os métodos mais freqüentemente usados em grupos populacionais de crianças e adolescentes normalmente são semelhantes ao empregados em estudos de adultos. Cada um dos métodos tem vantagens e limitações quando aplicado em crianças e adolescentes. Fundamentalmente todos os levantamentos dependem da motivação, confiança e habilidade dos sujeitos envolvidos e dos pais, em particular, para informarem acuradamente a ingestão alimentar habitual dos filhos. 41

Citado por Rockett e Colditz 38 um dos primeiros trabalhos sobre ingestão de nutrientes em crianças foi publicado em 1882 por Hasse, que estudou a dieta de crianças e adolescentes suíças e russas na faixa etária de dois a onze anos. Foi utilizado o método da pesagem de alimentos, analisando-se uma duplicata de cada alimento consumido quanto às quantidades de energia, proteína, carboidrato e gordura.

Posteriormente, na década de 40, Widdowson 42 observou que crianças britânicas estavam consumindo quantidades semelhantes de proteínas, gorduras e carboidratos, como havia sido relatado por Hasse 50 anos antes.

Rockett e Colditz ${ }^{38}$ consideraram esses estudos como precursores para, nas décadas seguintes, surgirem diversas pesquisas sobre dietas de crianças. Porém, o objetivo principal dessas pesquisas nutricionais era estabelecer as necessidades nutricionais para esse grupo populacional.

Paralelamente nos Estados Unidos, Burke37 desenvolveu em 1940 o "Método da história Dietética" para um estudo longitudinal sobre a saúde e o desenvolvimento da criança. O método de Burke para determinar a ingestão de um indivíduo envolveu o registro de hábitos dietéticos, a quantidade e freqüência de alimentos consumidos, bem como um recordatório 24 horas. Esse método foi amplamente utilizado em crianças e adolescentes por pesquisadores nos Estados Unidos e na Europa.

Nos anos 60, o estado nutricional de crianças e adolescentes americanas foi avaliado por meio do estudo "Teen state nutritional survey", que usou o método recordatório 24 horas para obtenção dos dados dietéticos. 43

Durante os anos 70, iniciaram-se nos Estados Unidos dois grandes estudos dietéticos: National Health and Nutritional Examination Survey (NHANES) e o Nationawide Food Comsumption Survey (NFCS) cujos dados, levantados pelo método recordatório 24 horas, demonstraram os hábitos alimentares das crianças. 44

Atualmente, métodos como o recordatório 24 horas, história dietética e registro de alimentos continuam sendo muito usados na avaliação dietética. Entretanto existem poucos estudos que indicam o "Questionário de freqüência de consumo alimentar para estudos em crianças".38

No caso de pré-escolares, as informações devem ser obtidas dos familiares, muito comumente, da mãe. Admite-se apenas uma pessoa para repassar as informações. Caso a informação seja obtida de um só entrevistado, maiores são as chances de as respostas serem mais completas. As evidências têm mostrado que, se as informações forem repassadas pela criança e o responsável, há uma tendência de superestimação. 45

A idade e a capacidade de resposta são importantes razões para designar diferentes métodos dietéticos de entrevista. Embora tenha havido considerável aumento na capacidade das crianças em responder questões sobre seu comportamento alimentar, expressar atitudes de decisão sobre o que comer e conceitos sobre nutrição antes de sete ou oito anos de idade, somente por volta dos 10 a 12 anos a criança tem capacidade de dar respostas sobre sua ingestão alimentar. Avaliação dietética em crianças é difícil, e os pesquisadores devem estar alertas para considerar as influências do ambiente 
sobre a criança, interferindo na sua escolha alimentar. 46

Segundo Fisberg et al.,5 o comportamento alimentar do adolescente está fortemente influenciado pelos hábitos alimentares e vinculado ao grupo etário a que pertence. Preocupações com a imagem corporal, hábitos e costumes do grupo têm características comuns. Omissão de refeição, consumo de alimentos altamente energéticos e pobres em nutrientes, consumo precoce de bebidas alcoólicas e tendências a restrições dietéticas são fatores que podem levar à obesidade e a anorexia nervosa. Todos esses fatores tendem a influenciar fortemente os registros alimentares desse grupo.

\section{Principais métodos dietéticos}

\section{Questionário de freqüência}

de consumo alimentar (QFCA)

Esse método tem sido citado por muitos autores como um dos principais instrumentos metodológicos para estudos epidemiológicos que relacionam a dieta à ocorrência de doença, por avaliar a ingestão alimentar de populações, ter boa reprodutibilidade e validade aceitável, além de ser mais prático, informativo, de fácil aplicação e de baixo custo. 4,6,47,48

Durante as décadas de 60 e 70, pesquisadores desenvolveram e utilizaram os Questionários de Freqüência de Consumo Alimentar em estudos de incidência de câncer. $\mathrm{Na}$ mesma época, investigadores britânicos estabeleceram as bases teóricas que fundamentaram esse método, defendendo a idéia de que o consumo de alimentos é determinado primeiramente pela freqüência. 47

Os primeiros questionários de freqüência alimentar, elaborados com maior rigor metodológico, datam do final da década de 60 e basearam-se num banco de dados que correspondia a sete dias de diário alimentar. 6

A construção de questionários pode ser feita a partir de um banco de dados de alimentos, que em geral é composto pelos alimentos e preparações mais freqüentemente consumidos pela população a ser estudada ou a partir de tabelas de composição de alimentos.

Salvo e Gimeno, 48 ao verificarem a reprodutibilidade e validade de um "Questionário de freqüência de consumo alimentar" em população com excesso de peso, previamente construíram um questionário a partir de um levantamento em prontuários de pacientes obesos. O QFCA foi desenvolvido para obter informação qualitativa e quantitativa sobre o padrão alimentar e a ingestão de alimentos ou nutri- entes específicos, sendo seu principal objetivo conhecer o consumo habitual de determinado grupo populacional.

A utilização do QFCA oferece vantagem na sua rapidez de aplicação, necessitando menos treinamento do entrevistador, uma vez que pode ser aplicado em entrevista, auto-administrado ou enviado pelo correio. Essa vantagem pode ser traduzida em menor custo e eficiência na prática epidemiológica. 49

Pereira e Koifman 50 analisaram 13 artigos, cujo critério de inclusão foi o uso do "Questionário de freqüência de consumo alimentar" em validação. Os autores concluíram que o QFCA foi um instrumento de grande utilidade no estudo do papel da dieta na etiologia das doenças crônicas.

Chiara e Sichieri51 desenvolveram um "Questionário de freqüência de consumo alimentar" simplificado para auto-avaliação em adolescentes, com alimentos associados ao risco de doenças coronarianas. Cerca de 650 adolescentes na "Pesquisa de Nutrição e Saúde do Rio de Janeiro", 1996, foram entrevistados para participarem da avaliação do QFCA simplificado. Após exclusões, 526 adolescentes participaram do estudo. O QFA contendo 80 itens de alimentos foi simplificado utilizando-se a equação proposta por Mensink e Katan (DCt), que permitiu reduzi-lo para 10 itens. Essa equação reflete a variação do colesterol sérico por influência da dieta. A inclusão de alimentos no "Questionário de freqüência de consumo alimentar" simplificado baseou-se nos alimentos que explicavam até $85 \%$ da variância total no nível de colesterol sérico por influência da dieta, bem como o valor calórico total da dieta, e que fossem fontes de ácidos graxos trans na alimentação habitual dos adolescentes. Estes autores sugeriram que, tendo conhecimento do seu padrão de consumo, os adolescentes poderiam buscar balancear sua dieta por meio de um questionário simplificado.

An e Shin 39 compararam dados antropométricos e ingestão de alimentos e nutrientes de adolescentes de áreas urbanas e ilhas de Kyungnam para identificar problemas nutricionais. Foi distribuído entre 383 adolescentes um "Questionário de freqüência de consumo alimentar", incluindo questões sobre ingestão dietética e situação socioeconômica. O peso e a altura foram medidos no início do estudo. Os resultados demonstraram que não houve diferença estatisticamente significante entre o peso dos estudantes de áreas urbanas e ilhas; o consumo de energia foi menor entre estudantes do sexo masculino das áreas urbanas, os quais tinham maior tendência a serem obesos, embora a ingestão de todos os nutrientes fosse muito mais baixa que a dos estudantes das ilhas. 
MacKeown et al.52 utilizaram um "Questionário semi-quantitativo de freqüência de consumo alimentar", em estudo de coorte longitudinal durante quatro anos para determinar a ingestão de energia, macro e micronutrientes em crianças de 5, 7, 9 e 10 anos de idade da África do Sul. Esses pesquisadores obtiveram as informações de parentes ou responsáveis pelas crianças e a escolha do método tomou como base um estudo anterior realizado em outra província da África do Sul, o qual demonstrou boa reprodutibilidade e validade para avaliar a ingestão dietética da população negra do país. Entretanto, ao discutir sobre a metodologia aplicada nesse estudo, os autores chamam a atenção de que, ingestão dietética é tão somente uma estimativa do consumo alimentar, não podendo ser avaliada sem erros. Os autores mostraram preocupação com a validação desse instrumento.

Estudando a possível validade e reprodutibilidade de um "Questionário de freqüência de consumo alimentar" para adolescentes do quarto e quinto períodos de uma escola americana, Domel et al. 53 compararam, semanal e mensalmente, um QFCA de frutas e vegetais com os alimentos registrados pelos adolescentes e não observaram nenhuma diferença significante entre a ingestão e as informações registradas nos questionários.

Nowak e Büttner, ${ }^{7}$ ao estudarem a relação entre tabus alimentares e o comportamento na ingestão alimentar em adolescentes australianos de quatro escolas privadas, utilizaram um "Questionário de freqüência de consumo alimentar" que também continha questões socioeconômicas, demográficas, atividades físicas, ambientais e comportamentais.

Os resultados demonstraram uma relação altamente significante entre crenças alimentares, consumo de alimentos, comportamento, ganho de peso e conhecimentos sobre nutrição.

Villar6 desenvolveu um "Questionário de freqüência de consumo alimentar" específico para adolescentes entre 14 e 19 anos de uma escola particular de Guarulhos, São Paulo. Foi elaborada uma lista de alimentos, identificando os alimentos de maior contribuição energética, a freqüência de consumo de alimentos foi sistematizada em unidades de tempo e foi estabelecido porcionamento de cada item alimentar. A lista de alimentos e as porções de alimentos referidas no instrumento foram elaboradas a partir de um banco de dados pertencente à outra pesquisa. Após definição da estrutura preliminar do QFCA, realizou-se um teste piloto, no qual o mesmo foi aplicado a 26 alunos de ambos os sexos na faixa etária de 14 a 17 anos (representavam $10 \%$ do total de alunos do segundo ano do ensino médio da escola). Esse piloto permitiu a avaliação do instrumento, o grau de exatidão do consumo alimentar e detecção de possíveis problemas.

Para Nowak e Büttner, 7 a associação de questionários permitiu associar algumas variáveis que interferem significativamente no consumo alimentar. Demonstraram que, mudanças de comportamento e associação de consumo alimentar com exercícios físicos são tarefas difíceis e complexas, que podem ser afetadas por fatores psicológicos, sociais, culturais, ambientais e comportamentais.

\section{Recordatório de 24 horas}

Consiste em obter informações escritas ou verbais sobre a ingestão alimentar das últimas 24 horas, com dados sobre os alimentos atualmente consumidos e informações sobre peso/tamanho das porções que deveriam ser, em tese, fornecidas por meio de fotografias ou modelos de porções.

O método recordatório 24 horas foi utilizado pela primeira vez no Brasil nos anos $30^{10} \mathrm{e}$, assim como o "Questionário de freqüência de consumo alimentar", foi desenvolvido por Burke com a finalidade de ensinar as mães a registrarem o consumo de alimentos dos filhos nas últimas 24 horas. 37

Bastante usado em todo o mundo, o método recordatório 24 horas é um instrumento de avaliação da ingestão de alimentos e nutrientes de indivíduos e grupos populacionais, mas requer um nutricionista ou entrevistador bem treinado para a realização da coleta de dados. Em geral, esse instrumento é bem aceito pelos entrevistados, o tempo de aplicação é curto, o custo é baixo e não promove alteração da dieta habitual. 20

No Brasil, esse método foi utilizado em grandes pesquisas como "Saúde e Nutrição das Crianças (NUPENS) da Universidade de São Paulo (USP), realizada em 1995 e 1996, que embasou os estudos de Monteiro e Conde. 54

Outra pesquisa foi o Estudo Multicêntrico de Salvador, "Condições de Vida, Saúde e Nutrição na Infância em Salvador", realizado por meio da parceria entre a Universidade Federal da Bahia com o Ministério da Saúde. Essa pesquisa identificou os padrões de saúde e nutrição de crianças menores de cinco anos da cidade de Salvador, Bahia. Dados sobre o padrão alimentar e adequação das dietas consumidas pelas crianças foram obtidos pela aplicação do recordatório 24 horas associado à freqüência alimentar. As informações foram obtidas das mães e agrupadas por faixa etária. Considerou-se o uso de álbum com desenhos de alimentos em suas dimensões normais e a utilização de medidas-padrão de 
líquidos, elementos facilitadores para auxiliar o entrevistado e aumentar o grau de confiabilidade das respostas. 55

A utilização do recordatório 24 horas em estudos epidemiológicos apresenta muitas vantagens, principalmente porque é rápido, relativamente barato e de fácil aplicação. Permite que a população estudada não seja alfabetizada e pouco altera o comportamento alimentar. Esse método avalia a dieta atual e estima valores absolutos ou relativos da ingestão de energia e nutrientes amplamente distribuídos no total de alimentos oferecidos ao indivíduo. ${ }^{4}$

Entretanto, o entrevistado tem que recordar, definir e quantificar sua ingestão alimentar do dia anterior à entrevista. Outras limitações importantes inerentes a esse método é que reflete a ingestão atual, não representando os hábitos alimentares. Também não permite considerar a sazonalidade, além do fato da ingestão real pode estar omitida pelo sub-registro. Dentre todas as limitações do recordatório 24 horas, a mais preocupante é que ele não fornece uma estimativa segura da absorção de nutrientes devido à variação do dia a dia e quando usado em estudos epidemiológicos, a habilidade de descrever relações significantes entre dieta e risco de doença será reduzida drasticamente. 2

Bingham 34 recomenda o emprego do método do recordatório 24 horas por três dias para avaliar o total da ingestão de alimentos e nutrientes, sendo esse método capaz de estimar as diferenças entre grupos de indivíduos em pesquisas epidemiológicas.

As informações obtidas por meio do recordatório 24 horas serão determinadas pela habilidade do indivíduo de recordar, a qual estará influenciada pelo sexo, idade e nível de escolaridade. A idade é o fator que mais influencia. Apesar de todas as limitações e significativas fontes de erro, esse método é muito usado. 3

Como o método requer informação detalhada sobre o consumo de alimentos mediante um relato, é necessária criteriosa preparação do desenho do estudo. Utilização de medidas caseiras, programas de computador, álbuns de fotografias devem fazer parte desta preparação detalhada. A única maneira de amenizar as fontes de erro (viés de memória, tamanho de medidas caseiras e estimação das porções) é associar ao recordatório 24 horas o uso de fotografias, réplicas de alimentos e kits com medidas caseiras, além de repetir a entrevista. 4,20

Quando o recordatório 24 horas é aplicado em crianças, é necessário que as perguntas possibilitem quantificar as sobras, para se saber exatamente as quantidades consumidas pela mesma. Avaliar o consumo alimentar de crianças através desse método requer a ajuda de um adulto, entretanto alguns autores sinalizam que a partir de 12 ou 13 anos, isso não se faz necessário. 46,56

Trigo, 17 comparando o método recordatório 24 horas com o de pesagem de alimentos, para verificar a validade do primeiro, encontrou baixa especificidade do recordatório 24 horas.

Baxter e Thompson,57 em estudo de análise retrospectiva, compararam a acurácia dos componentes e porções da alimentação escolar, pelo método recordatório 24 horas. Subdividiram os estudantes em dois grupos de estudo e os entrevistaram para obter dados sobre os componentes das refeições (Estudo 1) e as porções das refeições (Estudo 2). Os resultados mostraram erros de memória e inabilidade das crianças em informar os componentes e porções das refeições, em ambos os grupos, sendo maiores no Estudo 2. Esses resultados sugeriram que estudos validados são necessários para direcionar o desenvolvimento de protocolos de entrevistas, os quais maximizem a acurácia dos recordatórios dietéticos de crianças.

Segundo Eck et al.,58 alguns pesquisadores observam que, se a criança e seus pais combinam respostas no método recordatório 24 horas, a informação parece mais acurada que uma entrevista realizada apenas com um dos pais.

\section{Registro alimentar}

Esse método consiste em um indivíduo anotar em formulários previamente estruturados todos os alimentos e bebidas consumidas e suas respectivas quantidades durante determinado período, em geral ao longo de um dia. Caso o indivíduo seja orientado a anotar os seus dados de consumo logo após as refeições, o método poderá ser mais completo e preciso. Requer o uso de balança ou a ajuda de diferentes tamanhos de porções e a representação do que foi consumido em medidas caseiras tradicionalmente usadas.

Segundo Marr, 32 o registro alimentar que inclui o uso de balança para pesar os alimentos pode ser considerado um método de avaliação de ingestão bastante preciso, entretanto requer treinamento, esforço e muita colaboração por parte do entrevistado, fatores que fazem com que esse método seja pouco usado, principalmente em estudos populacionais.

Para Bonomo, 4 uma das principais vantagens do método é que ele independe de memória, sendo considerado o método mais válido para medir a ingestão alimentar. Entretanto, o autor concorda com Marr, ${ }^{32}$ reconhecendo que sua utilização envolve 
mais tempo do entrevistado, que deverá estar muito motivado, pois o resultado dependerá da sua cooperação. Além disso, os alimentos consumidos fora do lar também devem ser registrados.

Segundo Thompson e Byres, 59 a aplicação desse método não deve ultrapassar sete dias consecutivos, para não torná-lo insatisfatório devido à fadiga do entrevistado.

A principal desvantagem é o sub-registro alimentar ou omissão de alimentos, pois é muito difícil conhecer os ingredientes de uma preparação, principalmente quando essa é preparada e consumida fora do lar. Assim, as quantidades não refletirão com exatidão o hábito alimentar real. Esse método, como o recordatório 24 horas, recolhe informação sobre a ingestão atual de um indivíduo ou grupo populacional. As limitações mais relevantes são a impossibilidade de aplicar o método em pessoas não alfabetizadas, impaciência natural de crianças e adolescentes para preencher todos os formulários e seu alto custo.

\section{Pesagem de alimentos}

Esse método consiste no registro, pelo entrevistador, das quantidades de alimentos que efetivamente irão ser consumidas pelo entrevistado em medidas caseiras ou mediante pesagem direta dos alimentos, com auxílio de uma balança doméstica. 4

Ao descrever a metodologia de pesquisa aplicada ao ENDEF, Vasconcelos ${ }^{10}$ demonstra a notória interferência e possível influência do entrevistador sobre o indivíduo, que se constituem as maiores dificuldades no uso desse método: "... A metodologia consistia em visitar cada domicílio ao longo de sete dias consecutivos e o entrevistador pesava os alimentos que seriam consumidos na refeição seguinte, antes de sofrerem quaisquer alterações ou transformações, e deixar sacos plásticos para que fossem guardadas as sobras, para pesagem posterior " (2000: 20). 10

Cruz 60 em estudo de consumo alimentar de crianças entre um e seis anos de três creches da cidade de São Paulo, utilizou o método da pesagem direta para obter o peso médio das porções de alimentos oferecidas às crianças, dados de consumo alimentar e avaliar a concordância de dados de consumo. Com base nos resultados obtidos o autor relata que há concordância na comparação da pesagem direta de alimentos em nível individual e total, embora o individual seja mais preciso, e sugere que esse método possa ser usado com bons resultados para definir, em curto prazo, políticas de nutrição e saúde.

Embora seja considerado o mais exato para determinar a ingestão de alimentos, é um método mais demorado, oneroso, altamente invasivo e exige alto nível de cooperação das famílias e indivíduos.4,10

Klesge et al. 61 mediram a ingestão dietética de 30 crianças entre 24 e 48 meses durante 24 horas, utilizando dois métodos: o recordatório 24 horas, estimado pelo registro dos pais, habitualmente a mãe, e a pesagem direta de alimentos por um observador treinado que pesou todo o alimento preparado e o que não foi consumido. Os resultados obtidos pelo método recordatório 24 horas estavam altamente correlacionados com os encontrados pelo método da pesagem dos alimentos ingeridos.

\section{História dietética}

Este método consiste em uma associação de métodos em que, obrigatoriamente o "Questionário de freqüência de consumo alimentar" se associa com outro, que pode ser o recordatório 24 horas ou registro da pesagem, para verificar e validar os dados coletados inicialmente. Em geral utilizam-se três dias de registros alimentares por meio de medidas caseiras, observando, entre outras coisas, o tamanho da porção. Permite estimar a ingestão alimentar habitual, mas é desaconselhável para estudos populacionais, pois requer tempo para execução e seu custo é alto. 4

Fontanive et al. 40 utilizaram a história dietética incluindo um "Questionário de freqüência de consumo alimentar" em estudo de caso-controle para comparar o estado nutricional de adolescentes eutróficas com adolescentes com sobrepeso morando no Brasil. A história dietética demonstrou não haver diferença entre os grupos com relação à ingestão de nutrientes e energia. O "Questionário de freqüência de consumo alimentar" foi capaz de demonstrar que a ingestão de alimentos gordurosos era bem maior no grupo de adolescentes com sobrepeso que entre as eutróficas.

Para Domel et al.,53 instrumentos como o "Questionário de freqüência de consumo alimentar", recordatório 24 horas e registro de alimentos já foram usados para avaliar dietas de crianças, os quais mostraram-se muito mais desafiantes que avaliar dietas de adultos. Entretanto, a junção do QFCA com um dos métodos citados pode propiciar melhor interpretação dos resultados.

\section{Conclusões}

A medida correta de uma dieta constitui-se uma tarefa extremamente complexa e desafiadora para um epidemiologista nutricional, e, tratando-se de cri- 
anças e adolescentes, a tarefa torna-se mais complexa e de grande preocupação.

As dietas das crianças, como dos demais grupos populacionais, sofrem variações dia a dia, e seus hábitos alimentares podem mudar rapidamente. Crianças mais jovens, semelhantemente ao que freqüentemente ocorre com idosos, têm pouca habilidade para reportar, estimar e cooperar com os procedimentos de avaliação dietética, portanto muitas informações precisam ser substituídas pelas informações dos pais ou responsáveis.

Devido ao importante papel da dieta no crescimento, a possível relação entre dieta e doença durante a infância e adolescência, ao surgimento de doenças crônicas na idade adulta e ao aumento da obesidade entre os jovens de muitos países, pesquisadores, incansavelmente, devem investigar qual o melhor método para avaliar o consumo alimentar de crianças e adolescentes em estudos populacionais.

\section{Referências}

1. Buzzard IM. Rationale for an international conference series on dietary assessment methods. Am J Clin Nutr 1994; 59 Suppl: 143S-5S.

2. Margetts BM, Nelson M. Design concepts in nutritional epidemiology. 2 ed. New York: Oxford University Press; 1997.

3. Majem SLI. Tipos de estúdios em epidemiologia nutricional. In: Majem SLI, Bartrina JA, Verdú MJ. Nutrición y salud pública: métodos, bases científicas y aplicaciones. Barcelona: Masson; 1995.

4. Bonomo E. Como medir a ingestão alimentar? In: Dutra de Oliveira JE. Obesidade e anemia carencial na adolescência. São Paulo: Instituto Danone; 2000.

5. Fisberg M, Bandeira CRS, Bonilha EA, Halpern G, Hirschbruch MD. Hábitos alimentares na adolescência. Pediatr Mod 2000; 36: 766-70.

6. Villar BS. Desenvolvimento e validação de um questionário semi-quantitativo de freqüência alimentar para adolescentes [tese doutorado]. São Paulo: Faculdade de Saúde Pública da Universidade de São Paulo; 2001.

7. Nowak M, Büttner P. Relationship between adolescents food-related beliefs and food intake behaviors. Nutr Res 2003; 23: 45-55.

8. Lustosa TQO. Para que servem os dados sobre consumo alimentar? In: Consumo alimentar: grandes bases de informação. São Paulo: Instituto Danone; 2000.

9. Coimbra M, Meira JFP, Starling MBL. Comer e aprender: uma história da alimentação escolar no Brasil. In: Coimbra M. A alimentação escolar no Brasil: política e insti-
A definição do método dietético dependerá das características e dos objetivos do estudo, da população alvo, bem como, dos recursos disponíveis.

O caminho a percorrer é testar a validade e reprodutibilidade de métodos. Uma forma eficaz seria a comparação dos resultados de métodos indiretos com métodos diretos (exames bioquímicos).

Certamente, num futuro próximo, outros métodos surgirão para investigar o consumo alimentar de crianças, adolescentes, adultos e idosos, utilizando avançadas tecnologias disponíveis. Todavia, notório e relevante é não se desperdiçarem as importantes contribuições de pesquisadores como Bertha Burke e Walter Willett, da Universidade de Harvard, USA, Elizabeth Widdowson, Michael Nelson e Barrie Margetts, da Inglaterra; Josué de Castro, no Brasil e tantos outros, que, pioneiramente, iniciaram as pesquisas sobre estimativas da ingestão dietética de indivíduos e grupos populacionais. tuição. Belo Horizonte: Universidade Federal de Minas Gerais; 1982.

10. Vasconcelos M. Caracterização geral e principais aspectos metodológicos do ENDEF - Estudo Nacional de Despesas Familiares. In: Consumo alimentar: grandes bases de informação. São Paulo: Instituto Danone; 2000

11. Schaefer EJ, Augussin MMS, Rasmussen H, Ordovas JMO, Dwyer JT. Lack of efficacy of a food-frequency questionnaire in assessing dietary macronutrient intakes in subjects consuming diets of know composition. Am J Clin Nutr 2000; 71:746-51.

12. López JV. Validez de la evaluación de la ingesta dietética. In: Majem LS, Bartrina JA, Verdú JM. Nutrición y salud pública. Métodos, bases científicas y aplicaciones. Barcelona: Masson; 1995.

13. Block G, Hartman AM. Issues in reproducibility and validity of dietary studies. Am J Clin Nutr 1989; 50: 1133-38.

14. Willet WC, Buzzard IM. Foods and nutrients. In: Willett W. Nutritional epidemiology. New York: Oxford University Press; 1998. v. 2. p. 18-32.

15. Dwyer JT. Dietary assessment. In: Shills ME, Olson JA, Shike M. Modern nutrition health and disease. 9. ed. Philadelphia: Lea \& Febiger; 1999, 937-59.

16. Willett WC. Nutricional epidemiology. New York: Oxford University Press, 1998.

17. Trigo M. Estudo da metodologia de inquérito dietético: validade de método recordatório de 24 horas [tese doutorado]. São Paulo: Faculdade de Saúde Pública da Universidade de São Paulo; 1993. 
18. Farnsworth H. Defects, uses and abuses of national food supply and consumption data: Food Research Institute Studies. Stanford: Stanford University; 1961.

19. Vasconcelos FG. Como nasceram meus anjos brancos. A constituição do campo da nutrição em saúde pública em Pernambuco. Recife: Bagaço; 2001.

20. Salvo VLMA, Gimeno SGA. Métodos de investigação do consumo alimentar: entrevista. 2003. Disponível em: http://www.nutricaoempauta.com.br [2003 nov 11].

21. Galeazzi MA. Aplicações e limitações ao uso das pesquisas de orçamentos familiares. In: Consumo alimentar: grandes bases de informação. São Paulo: Instituto Danone; 2000.

22. IBGE (Fundação Instituto Brasileiro de Geografia e Estatística). Estudo nacional de despesa familiar, ENDEF. Rio de Janeiro: O Instituto; 1976.

23. INAN (Instituto Nacional de Alimentação e Nutrição). Pesquisa Nacional sobre Saúde e Nutrição: perfil de crescimento da população brasileira de 0 a 25 anos. Brasília (DF): O Instituto; 1990.

24. Galeazzi MAM, Domene SMA, Sichieri R. Estudo multicêntrico sobre consumo alimentar. Brasília (DF): Ministério da Saúde; 1997.

25. Carvalho CMRG, Nogueira AMT, Teles JBM, Paz SMR, Sousa RML. Consumo alimentar de adolescentes matriculados em um colégio particular de Teresina, Piauí, Brasil. Rev Nutr 2001; 14: 85-93.

26. Kapazi BM, Di Pietro PF, Avancini SRP, Freitas SFT, Tramonte VLCG. Consumo de energia e macronutrientes por adolescentes de escolas públicas e privadas. Rev Nutr 2001; 14 Supl: 27-33.

27. Vieira VCR, Priore SE, Ribeiro SMR, Franceschini SCC, ALMEIDA LP. Perfil socioeconômico, nutricional e de saúde de adolescentes recém ingressos em uma universidade pública brasileira. Rev Nutr 2002; 15: 273-82.

28. Spinelli MGN, Goulart RMM, Santos ALP, Gumiero LDC, Farhud CC, Freitas EB, Dantas LF. Consumo alimentar de crianças de 6 a 18 meses em creches. Rev Nutr 2003; 16: 409-14.

29. Garcia GCB, Gambardella AMD, Frutuoso MFP. Estado nutricional e consumo alimentar de adolescentes de um centro de juventude da cidade de São Paulo. Rev Nutr 2003; 16: 41-50.

30. Rotenberg S, De Vargas S. Práticas alimentares e o cuidado da saúde: da alimentação da criança à alimentação da família. Rev Bras Saúde Matern Infant 2004; 4: 85-94.

31. Gibson RS. Principles of nutritional assessment. Oxford: Oxford University Press; 1990.

32. Marr JW. Dietary surveys: purposes and methods. World Rev Nutr Diet 1971; 13: 105-64.

33. Cintra IP, Von Der Heyde MED, Schimitz BAS, Franceschini SCC, Taddei JA, Sigulem DM. Métodos de inquéritos dietéticos. Cad Nutr 1997; 13: 11-23.

34. Bingham SA. The dietary assessment of individuals: methods, accuracy, new thechniques and recommendations. Nutr Abstr Rev 1987; 57: 705-42.

35. Block G, Hartman AM. Issues in reproducibility and validity of dietary studies. Am J Clin Nutr 1989; 50: 1133-8.

36. Willett WC. Diet and nutrition. In: Schottenfeld D, Fraumeni JF. Cancer epidemiology and prevention. 2nd.ed. Oxford: Oxford University Press; 1996.

37. Burke B. The dietary history as a tool in research. J Am Diet Assoc 1947; 23: 1041-6.
38. Rockett HRH, Colditz GA. Assessing diets of children and adolescents. Am J Clin Nutr 1997; 65 Suppl: 1116S-22S.

39. An GS, Shin DS. A comparison of the nutrient intake of adolescents between urban areas and islands in south Kyungnam. Korean J Comm Nutr 2001; 6: 271-81.

40. Fontanive RS, Costa RS, Soares EA. Comparison between the nutricional status of eutrophic and overweight adolescents living in Brazil. Nutr Res 2002; 22: 667-78.

41. FAO (Food and Agriculture Organization), WHO (World Health Organization), UNU (Universidade das Nações Unidas). Energy and protein requirements. Geneve: WHO; 1985. (Techinical Report Series, 724)

42. Widdowson EM. A study of individual children's diets. Part I. J Hyg 1936; 36: 269-92.

43. Rockett HR, Wolf AM, Colditz GA. Development and reproducibility of a food frequency questionnaire to assess diets of children and adolescents. J Am Diet Assoc 1995; 95: 336-40.

44. CDC (Center for Disease Control). Growth charts for the United States: methods and development. Vital Health Statistics Series 2000; 11: 1-190.

45. Baranovski T, Sprague D, Baranovski JH, Harrison JA. Accuracy of maternal dietary recall for preschool children. J Am. Diet. Assoc 1991; 91: 669-74.

46. Frank GC. Environmental influences on methods used to collect dietary data from children. Am J Clin Nutr 1994; 59 Suppl: 207S-11S.

47. Willett WC. Future directions in the development of food-frequency questionnaires. Am J Clin Nutr 1994; 59 (Suppl): 171S-4S

48. Salvo VLMA, Gimeno SGA. Reprodutibilidade e validade do questionário de freqüência de consumo alimentar. Rev Saúde Pública 2002; 36: 505-12.

49. Sichieri R, Everhart JE. Validity of a Brazilian food frequency questionary against dietary recalls and estimated energy intake. Nutr Res 1998; 18: 1649-59.

50. Pereira RA, Koifman S. Uso do questionário de freqüência na avaliação do consumo alimentar pregresso. Rev Saúde Pública 1999; 33: 610-21.

51. Chiara VL, Sichieri R. Consumo alimentar em adolescentes. Questionário simplificado para avaliar o risco cardiovascular. Arq Bras Cardiol 2000; 77: 332-6.

52. Mackeown JM, Cleaton-Jones PE, Norris SA. Nutrient intake among a longitudinal group of urban black South African children at four interceptions between 1995 e 2000. Nutr Res 2003; 23: 185-97.

53. Domel SB, Baranowski T, Leonard SB, Davis H, Riley P, Baranowski J. Accuracy of fourth and fifth-grade students'food records compared with school-lunch observations. Am J Clin Nutr 1994; 59 Suppl: 218S-20S.

54. Monteiro CA, Conde WL. Tendência secular da desnutrição e da obesidade na infância na cidade de São Paulo (1974 - 1996). Rev Saúde Pública 2000; 34 Supl: 52-61.

55. Assis AMO, Barreto ML, Santos MLP, Sampaio LR, Magalhães LP, Prado MS, Santos, NS, Galvão NMS, Silva RCR, Oliveira VA Condições de vida, saúde e nutrição na infância em Salvador. Salvador: Universidade Federal da Bahia, Instituto de Saúde Coletiva, Ministério da Saúde; 2000.

56. Baxter SD, Smith AF, Guinn CH, Thompson WO, Litaker MS, Baglio ML, Shaffer NM, Frye FHA. Interview format influences the accuracy of children's dietary recalls 
validated with observations. Nutr Res 2003; 23: 153746.

57. Baxter SD, Thompson WO. Accuracy by meal component of fourth-graders'school lunch recalls is less when obtained during a 24 -hour recall than as a single meal. Nutr Res 2002; 22: 679-84

58. Eck IH, Klesges RC, Hanson CL. Recall of a child's intake from one meal: are parents accurate? Am Diet Assoc 1989; 89: 669-74.

59. Thompson FE, Byers T. Dietary assessment resource manual. J Nutr 1994; 124 Suppl: 2245S-2317S.

Recebido em 11 de Janeiro de 2003

Versão final apresentada em 7 de maio de 2004

Aprovado em 12 de julho de 2004
60. Cruz ATR. O consumo alimentar de crianças: avaliação pelo "método da pesagem direta" em três creches no Município de São Paulo-SP [dissertação mestrado]. São Paulo: Faculdade de Ciências Farmacêuticas da Universidade de São Paulo; 2001.

61. Klesges RC, Klesges LM, Brown G, Frank GC. Validation of the 24 hour dietary recall in preschool children. J Am Diet Assoc 1987; (10): 1383-5. 